\title{
Design and Implementation of Torque Sensing System of Intelligent E-bike
}

\author{
Xiadong Zhang ${ }^{1, \text { a }}$, Yunfei $\mathrm{Li}^{1, \mathrm{~b}}$ and Juncheng Jia ${ }^{1, \mathrm{c}}$ \\ ${ }^{1}$ School of Computer Science and Technology, Soochow University, Suzhou 215000, China; \\ a515246437@qq.com
}

Keywords: E-bike, torque sensor, ICPT, wireless transmission.

\begin{abstract}
Aiming at the difficulties in developments, high cost of torque sensing system of e-bike, a novel torque sensing system based on sensitive sensing component has been proposed. The signal acquisition component which includes a torque sensor based on a sensitive strain gauge, an electrical signal processing unit built up with differential amplifier and $\mathrm{A} / \mathrm{D}$ conversion circuit, and a computation and wireless transceiver center based on lower power consumption processor CC1110 is installed on the middle axle of e-bike to achieve signal acquisition. Moving weighted average filtering algorithm is applied in the software to enhance the stability of data acquisition. The methods of least-square is used to ensure the high-precision of data acquisition. The wireless transmission of power and signal is realized by using the technology of inductive power which based on inductive coupled power transfer (ICPT) and technology of digital wireless communication. Focused on accuracy and durability, the system is tested based on simulating the actual environment. The experimental results indicate that the system is stable, high precision and the error is less than 1\% within the whole measurement in range of $0 \sim 20 \mathrm{~km}$. The work in this paper has practical value.
\end{abstract}

\section{Introduction}

Comparing with the traditional electric bicycle, the intelligent electric-assisted bicycle (E-bike) of lithium battery shows significant advantages. The speed of the traditional electric bicycle is controlled by the speed-regulating hand, while the E-bike provides motor assist via sensing the real-time torque pushed on the pedal. The E-bike makes the "intelligent riding" come true. With the increment of consciousness for environmental protection and energy-saving, the traditional one would be replaced by the E-bike in the future. As a core system of sensing the riding state, torque sensing system is the key component of the E-bike. Recently, the research of E-bike still stays in the initial stage [1]. Most of the torque sensing system is based on hall sensor. However, some shortcomings, such as complicated mechanical construction and high requirement of installation exist in it. In some foreign countries, the E-bike has occupied a certain market. Due to the prime cost and technological requirements, it is difficult to be popularized in domestic. For example, the torque sensing system with simple structure and strong reliability using torque sensor based on inverse magneto-elastic effect has been developed by BOSCH in Germany. However, the application and industrialization of inverse magneto-elastic material is in the initial stage in home [2]. Therefore, it is hardly to be popularized in the E-bike. Some scholars suggest that some torque sensors like Chain Wheel, Crankshaft and Back Hook could be used in torque sensing system of the E-bike. However, those kinds of sensors are exposed to the outside environment, which may result in poor stability [3].

In this paper, a new torque sensing system is proposed based on the research and analysis of the existing torque sensing system of the E-bike. The sensitive sensing material, inductive coupled electric transfer and technology of digital wireless communication are combined, which shows high stability, accuracy and long-term durability. 


\section{Design of Overall System}

Figure 1 shows the two modules of the overall system: one is called torque acquisition module to collect the real-time torque signal. It is placed in the pedal wheel bearing of the E-bike and rotates with the axis. And the other is called torque receiving module to receive the torque signal, which is fixed under the crankset to connect with the motor controller.

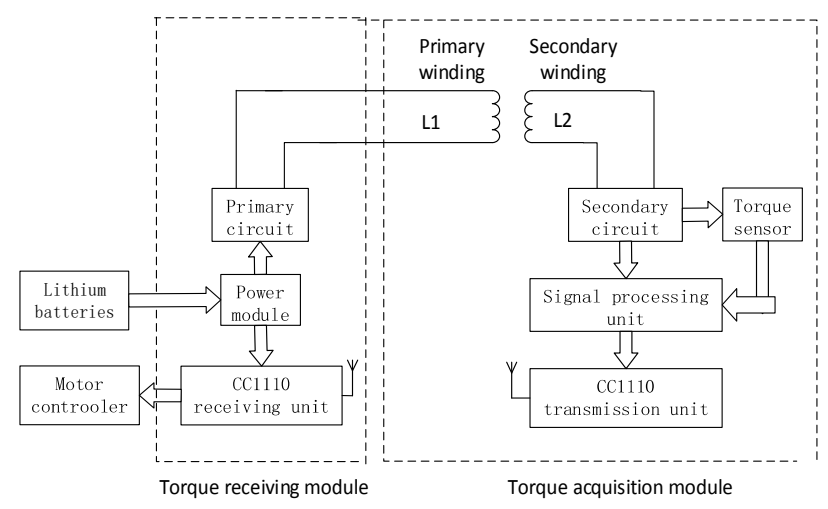

Figure 1. Diagram of the overall system.

Since the torque acquisition module is in a rotating state, therefor, the wireless transmission of power and signal is realized by using the technology of inductive coupled power transfer and digital wireless communication. The torque signal is sent to the receiving module by CC1110 wireless transmission unit, while the receiving module use inductive coupling model to provide energy for the acquisition module. Motor controller controls the output power of the motor to achieve intelligent electric assistance according to the value of torque signal.

\section{Design of Hardware Circuit}

According to the requirement of the system, secondary circuit, torque sensor, signal processing unit and CC1110 wireless transmission unit are included in the torque acquisition module. Power supply module, primary circuit and CC1110 wireless receiving unit are included in the torque receiving module.

\subsection{Wireless Power Supply Unit}

The power supply of the torque receiving module is provided by the lithium batteries with $36 \mathrm{~V} \mathrm{DC}$ power supply. The LM2576 is selected to convert the $36 \mathrm{~V}$ DC into a stable $5 \mathrm{~V}$ voltage to provide working voltage for the torque receiving module.

The torque receiving module transfers the electrical energy for the torque acquisition module with the form of electromagnetic induction coupling model. The principle of the inductive coupling power supply can be explained as follow: first, the primary winding is driven via the high frequency alternating current to produce a high frequency variations electromagnetic field in the surrounding spaces. Second, due to the changes of the magnetic flux, the secondary winding in this electromagnetic field generates induced electromotive force. Finally, a certain load capacity of DC is obtained after commutating and voltage-stabilizing.

Comprehensive consideration of mechanical structure, size and volume of the system, as well as the requirements of working voltage, current, the primary circuit and secondary circuit of designed wireless power supply unit are shown in Figure 2.

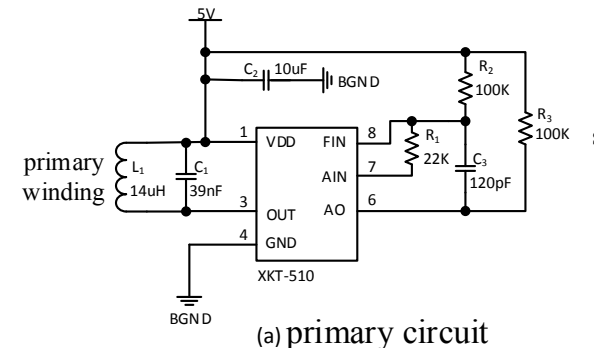

(a) primary circuit

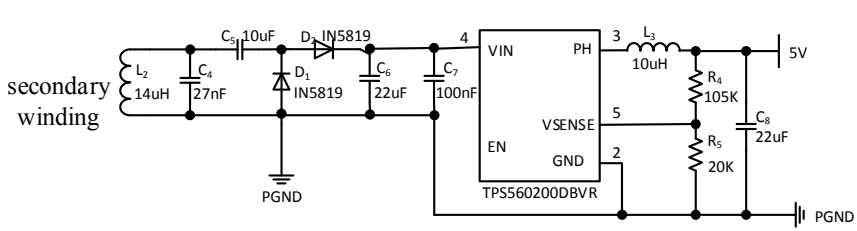

(b) secondary circuit 
Figure 2. Wireless power supply unit.

The square wave modulation generator integrated chips XKT-510 and voltage regulator TPS560200 are adopted in this paper. The power output pin OUT of XTK-510 produces $200 \mathrm{kHz}$ frequency of the square wave signals. The LC oscillation generates $200 \mathrm{kHz}$ of high frequency alternating current in primary winding. And the induced alternating current of secondary winding is sent into voltage chip TPS560200 as input, while output of the 5V DC is the power supply for the operation of torque acquisition module. In order to reduce power loss and improve the efficiency of power transmission, the primary side parallel-secondary side parallel compensation (PP) mode is used for the resonance compensation [4]. The primary winding is winded on outer framework of axis and holds still. Secondary side winding is winded in inner framework of axis, and rotates with the mean axis. The value of inductances for the primary winding and the secondary winding is $14 \mathrm{uH}$. The outer framework of axis is nested in inner framework to simplify the structure and guarantee high reliability, as well as protecting from any effects to rotate parts in the assembly and disassembly process.

Experimental result indicate that with the supply voltage of $5 \mathrm{~V}$, the secondary circuit can provide the highest value of $90 \mathrm{~mA}$, which fully meet the power supply demand of torque acquisition module.

\subsection{Design of Torque Sensor.}

The mechanical structure of the bicycle shows that the exerted torque is first transmitted from left/right side pedals to the crank shaft when the rider rides the bicycle. And then transmitted to the axis. With those two steps, the torque can produce tiny deformation on the axis. In this paper, FCT-2-350-11 model of resistance strain gauge is selected. Four strain gauges are pasted on the same cross-section of specified position on the axis. The angles between the strain gauges and the axis are $45^{\circ}$ and $135^{\circ}$, respectively. Then a Wheatstone bridge is built up. The change rate $\Delta R / R$ of the bridge resistance is proportional to the torque of the axis after the torque exerted on axis changes. And a tiny stress change on the axis can result in the change of the balance status of the bridge. As a result, the amount of the resistance change can be converted into $\mathrm{mV}$ level of differential voltage signals by Wheatstone bridge.

\subsection{Design of Signal Processing Unit.}

Since the output signal of torque sensor is $\mathrm{mV}$ level and could not be recognized directly by the CC1100 wireless transmission unit, the signal amplification circuit and A/D conversion circuit should be incorporated. The combined amplification and A/D conversion circuit is shown in Figure 3.

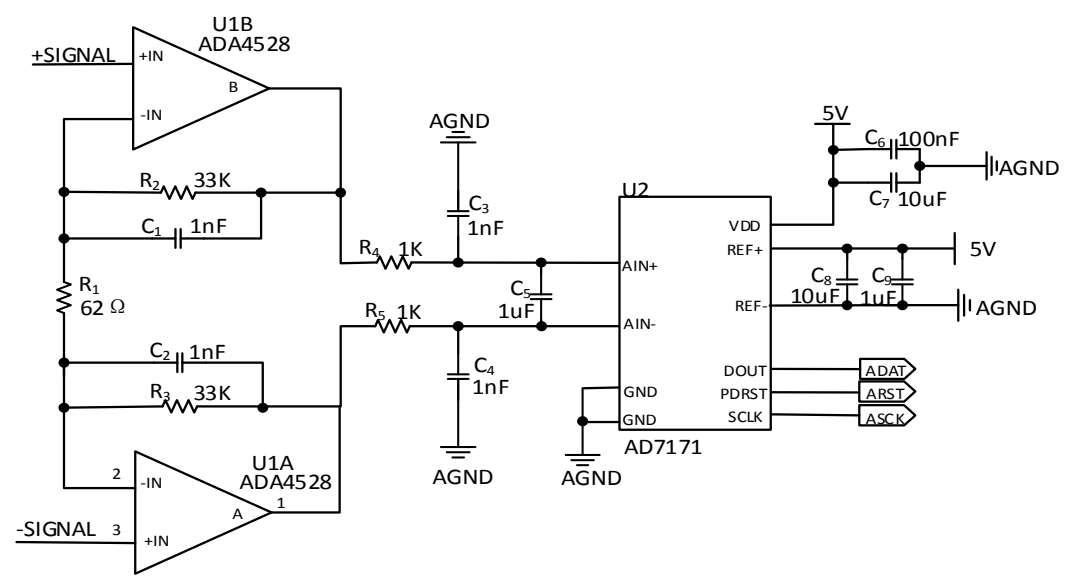

Figure 3. Signal processing unit.

As shown in Figure 3, the ADA4528 integrated chip is selected as operation amplifier. The output signal of torque sensor is processed by Different Amplification, which can restrain the effects of the common-mode signal and enhance the accuracy of data. The enlargement factor A is set to be 107 to achieve the accuracy of the output signal. The enlargement factor is expressed as follow, 


$$
A=2 \times \frac{R_{2}}{R_{1}}+1=2 \times \frac{33000}{62}+1=107
$$

The AD7171 chip integrated a 16-bits analog-digital converter is adopted in the A/D conversion circuit. To collect analog signal, the voltage signal amplified by the amplification circuit is transmitted into the AD7171. The output code of the AD7171 with the form of Shift Binary Coding communicates with the CC1110 via the data-output pin DOUT, clock pin SCLK and reset pin PDRST. The output code can be expressed as follow,

$$
\text { Code }=2^{N-1} \times\left(\frac{V_{I N X}}{V_{R E F}}+1\right)
$$

where VINX is the input voltage, and VREF is the reference voltage. Since the AD7171 is a 16-bits analog-digital converter, thus, $\mathrm{N}$ is set to be 16 .

\subsection{Design of Wireless Transceiver Unit.}

The CC1110 microcontroller with lower power consumption and 433MHz transceivers is selected as the core component in the wireless transceiver unit. Benefits of using the CC1110 include tighter protocol timing, security and improved performance. CC1110 processes the RF data stored in the RF register via the DMA (Directional Memory Access) module without the CPU, which alleviates the load of CPU.

\section{Design of System Software}

\subsection{Method and Implementation of Nonlinearity Rectification}

Since the relationship between the input and output of the torque sensor is nonlinear, so it needs to be rectified by the software. The curve fitting algorithm is incorporated to achieve nonlinearity rectifying in this paper. The relationship between the output voltage $U$ of the Wheatstone bridge and the torque $\mathrm{M}$ exerted on the pedal can be express as follow,

$$
U=f(M)
$$

The anti-function can be obtained by the least-square, which can be explained as follow,

$$
M=f^{-1}(U)
$$

Second-order polynomial is used in the anti-function to satisfy the processing capacity of microprocessor and precision requirement of data, which can be shown as,

$$
M=a_{0}+a_{1} U^{1}+a_{2} U^{2}
$$

The parameter a0, a1, a2 is stored in the FLASH of the microprocessor. The value of torque can be calculated by (5) after the voltage signal is obtained.

\subsection{Design of CC1110 Wireless Transmission Unit}

The diagram of software design for the CC1110 Wireless transmission unit is shown in Figure 4. The system will enter into the subroutine of torque collecting, processing, and wireless emission after it is initialized.

In this paper, to guarantee the reliability and accuracy of the measurement, the weighted average filtering algorithm is incorporated after each $\mathrm{A} / \mathrm{D}$ conversion. The window of the algorithm is set to be 10, and the weights are put in the array stored in the storage area. The data which center to the current moment have higher weights. The filtered data within the window is the output value of the filter. The output can be calculated as follow,

$$
U_{\text {out }}=\frac{1}{10} \sum_{i=1}^{10} C_{i} U_{i}
$$

where Uout represents the output of the filter. $\mathrm{Ci}$ is the weights, and Ui represents the $\mathrm{i}$-th samples.

To realize the nonlinearity rectification, we put the data filtered into (5) and obtain the corresponding torque value. The value is sent to the RF register by the DMA mode for wireless transmission. 
Due to the actual processing capacity of the microprocessor, the working frequency of the wireless transmission unit is set to be $20 \mathrm{~Hz}$.

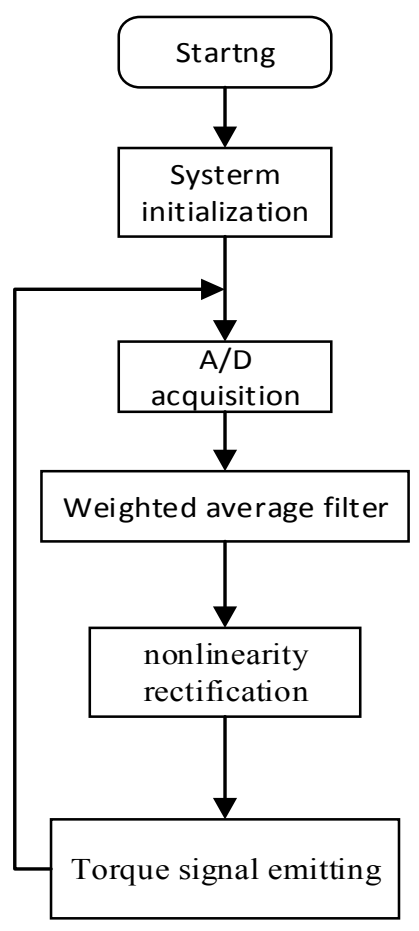

Figure 4. The software diagram of transmission unit

\subsection{Design of CC1110 Wireless Receiving unit}

The CC1110 wireless receiving unit enters into the RF receiving mode after it is initialized. After receiving the wireless data, the microprocessor will check whether the source address and the destination address of the data are identical with those set in transmission unit. If they are not consistent, the data will be discard directly, otherwise the value of the data will be judged. If the range of the torque value is between $0 \sim 95 \mathrm{Nm}$, the data will be sent to the motor controller via a serial port, otherwise the variable "ErrorCount" will be accumulated 1. If the value of the "ErrorCount" exceeds the threshold, the error message will be sent to motor controller to alert user to inspect the system in a timely manner.

\section{Testing Methods and Results}

In order to test the performance and feasibility in practical application of the system, a set of test platform based on the Lab-VIEW is developed. Figure 5 shows the hardware system of the test platform. It includes gear and chain, servo motor, standard torque sensor and magnetic powder brake. The servo motor drives gear and chain to simulate the actual torque during riding. The magnetic powder brake provides the load to simulate the resistance. The standard torque sensor is used to provide standard torque to compare with the measured torque signal. Aiming at the accuracy of the measurement and the durability of the system, the test method are described as follow.

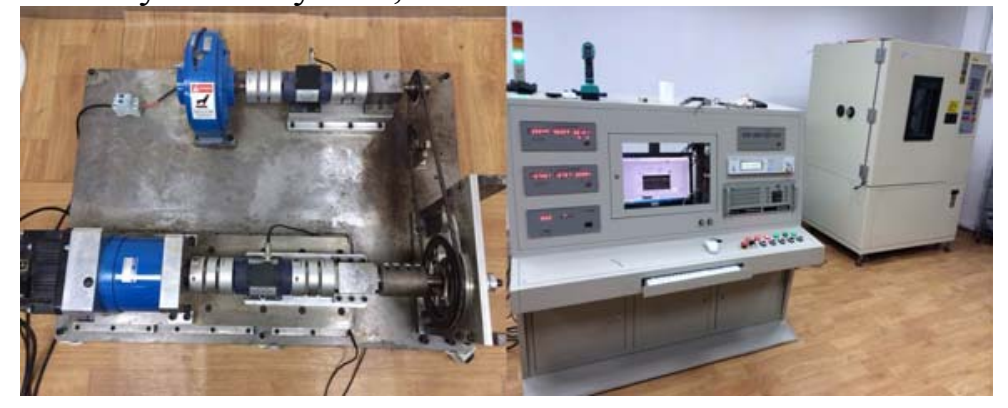

Figure 5. The hardware system of the test platform 


\subsection{Test Methods of Accuracy and Durability}

In the process of riding, the torque exerted on the axle changes periodically, and the change consistent with the sinusoidal wave. To be consistent with the reality riding process, the range of the sinusoidal wave is set to be $0 \sim 95 \mathrm{Nm}$, and the riding speed is $20 \mathrm{Km} / \mathrm{h}$. Torque receiving module is fixed under the chain wheel, and transmits the real-time signal to the industrial computer. The Lab-VIEW software deals with the data from the torque receiving module and displays them on the interface. Then, the full scale error $(\delta)$ is calculated as follow,

$$
\delta=\frac{\operatorname{MAX}\left(\sum_{i=1}^{M} \mid m_{i}-m_{i}^{\prime}\right)}{95} \times 100 \%
$$

where $M$ represents the number of sample points (20 points are sampled within one period in this paper). ${ }^{m}$ represents the $\mathrm{i}$-th standard value, and $m_{i}^{\prime}$ represents the i-th testing value.

\subsection{Testing Results}

A $20000 \mathrm{Km}$ cumulant is a complete testing cycle. Each test point is obtained after operating $100 \mathrm{Km}$. The relationship between the test mileage and the full scale error can be obtained. Since the attenuation of the resistance strain gauge, the accuracy of the system shows a slight decline with the increment of the testing mileage. The range of the full scale error is $0.3 \% \sim 0.8 \% \mathrm{FS}$ within $20000 \mathrm{Km}$, which fully meet the actual demand.

\section{Conclusion}

In this paper, a newly E-bike torque sensing system is designed and implemented. In this new system, the wireless power supply, the strain gauge sensor and the wireless digital communication are combined, and the compensation capability of the microprocessor is utilized. Due to the complex operation environment of the E-bike, the torque acquisition module is hidden in the axle to avoid the influence of the water and dust. The test results indicate that the system works well with high stability and accuracy. On the other hand, it satisfies the application requirement and provides a technical selection for the development and improvement of the E-bike.

\section{ACKNOWLEDGMENT}

This work is supported in part by National Natural Science Foundation of China (61272449), Joint Innovation Funding of Jiangsu Province (BY2014059-02), and Collaborative Innovation Center of Novel Software Technology and Industrialization.

\section{References}

[1]. Information on: http://en.wikipedia.org/Wikipedia.org/wiki/Electric_bicycle.

[2]. Information on: http://www.idbike.com/tmm-powermanagement.htm.

[3]. Information on: http://www.thun.de/en/products/sensor-technology.

[4]. INAGAKIN. Theory of image impedance matching for inductively coupled power transfer syste. Microwave Theory and Techniques. Vol.62(2014) No.4,p.901-908 